\title{
Assessment of genetic risk for improved clinical-neuropathological correlations
}

\author{
Barbara E. Spencer ${ }^{1}$, Robin G. Jennings ${ }^{1}$, Chun C. Fan ${ }^{2,3}$ and James B. Brewer ${ }^{1,3^{*}}$
}

\begin{abstract}
In the clinical diagnosis of dementia with Lewy bodies, distinction from Alzheimer's disease is suboptimal and complicated by shared genetic risk factors and frequent co-pathology. In the present study we tested the ability of polygenic scores for Alzheimer's disease, dementia with Lewy bodies, and Parkinson's disease to differentiate individuals in a 2713-participant, pathologically defined sample. A dementia with Lewy bodies polygenic score that excluded apolipoprotein E due to its overlap with Alzheimer's disease risk was specifically associated with at least limbic (transitional) Lewy-related pathology and a pathological diagnosis of dementia with Lewy bodies. An Alzheimer's disease polygenic score was associated with neuritic plaques and neurofibrillary tangles but not Lewy-related pathology, and was most strongly associated with an Alzheimer's pathological diagnosis. Our results indicate that an assessment of genetic risk may be useful to clinically distinguish between Alzheimer's disease and dementia with Lewy bodies. Notably, we found no association with a Parkinson's disease polygenic score, which aligns with evidence that dementia with Lewy bodies has a distinct genetic signature that can be exploited to improve clinical diagnoses.
\end{abstract}

Keywords: Alzheimer's disease, Dementia with Lewy bodies, Parkinson's disease, Polygenic risk, Diagnosis

\section{Introduction}

In heterogeneous disease cohorts, accurate distinctions between Alzheimer's disease (AD) and related dementias may improve precision in care delivery and thus lead to better outcomes. In the diagnosis of dementia with Lewy bodies (DLB), distinction from AD is suboptimal and complicated by the frequent co-occurrence of $\mathrm{AD}$ neuropathologic changes (NC) with Lewy body (LB) pathology. Patients clinically diagnosed with AD often present with concurrent LB pathology at autopsy, though many studies have attempted to tease apart the differences in clinical presentations to better reflect underlying pathology $[2,6,9,14,21,25]$.

Incorporating information about genetic risk into a difficult differential diagnosis may improve clinicalneuropathological correlations. A recently developed

\footnotetext{
*Correspondence: jbrewer@ucsd.edu

${ }^{1}$ Department of Neurosciences, University of California, San Diego, 9500 Gilman Drive, Mail Code 0949, La Jolla, CA 92093, USA

Full list of author information is available at the end of the article
}

AD polygenic hazard score (PHS) is associated with the hallmark Alzheimer's disease neuropathologic changes $(\mathrm{ADNC})$, neuritic plaques and neurofibrillary tangles. However, the large-scale genetic studies that identify such risk typically rely on clinical diagnoses, which are imperfect proxies for the often mixed underlying pathologies $[5,20]$. While the AD PHS has reported associations with LBs [22], it is unclear whether this reflects a shared genetic risk between pathologies, a byproduct of the common presence of $\mathrm{LB}$ co-pathology with $\mathrm{AD}$, since the level of ADNC was not controlled for in the analysis, or a lack of specificity in the AD PHS due to the presence of LBs or other mixed pathologies in those clinically diagnosed with AD.

DLB has both clinical features and genetic risk factors that overlap with both $\mathrm{AD}$ and Parkinson's disease (PD). The apolipoprotein $\mathrm{E}(A P O E) \varepsilon 4$ allele is the strongest genetic risk factor for late onset $\mathrm{AD}$ and is also overrepresented in pure DLB and PD with dementia [24]. The $S N C A, G B A$, and $B C L 7 C / S T X 1 B$ genes are implicated in risk for both DLB and PD $[8,16]$, though the associations

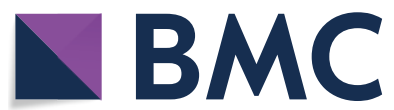

(c) The Author(s). 2020 Open Access This article is licensed under a Creative Commons Attribution 4.0 International License, which permits use, sharing, adaptation, distribution and reproduction in any medium or format, as long as you give appropriate credit to the original author(s) and the source, provide a link to the Creative Commons licence, and indicate if changes were made. The images or other third party material in this article are included in the article's Creative Commons licence, unless indicated otherwise in a credit line to the material. If material is not included in the article's Creative Commons licence and your intended use is not permitted by statutory regulation or exceeds the permitted use, you will need to obtain permission directly from the copyright holder. To view a copy of this licence, visit http://creativecommons.org/licenses/by/4.0/. The Creative Commons Public Domain Dedication waiver (http://creativeco mmons.org/publicdomain/zero/1.0/) applies to the data made available in this article, unless otherwise stated in a credit line to the data. 
at the $S N C A$ locus differ between the two $[1,8]$. Given the relatively low accuracy of a DLB diagnosis, genetic studies typically examine relatively small, neuropathologically confirmed DLB cohorts $[8,19]$, with few genome-wide significant variants identified. Then, genetic risk for PD, discovered in well-powered studies, may be better suited to predict the underlying LB pathology in DLB cases. In a pathologically defined cohort we tested the hypothesis that the AD PHS, a DLB polygenic risk score (PRS), and a PD PRS can differentiate individuals with DLB from those who have AD.

\section{Methods \\ Participants}

A sample of 437 participants was selected from the Shiley-Marcos Alzheimer's Disease Research Center (ADRC) of the University of California, San Diego (UCSD). An independent sample of 3982 participants evaluated at other ADRCs was selected from the National Alzheimer's Coordinating Center (NACC). Inclusion was limited to participants who had undergone genotyping and a neuropathological assessment at autopsy. All data were collected through the NACC uniform data set, minimum data set, or neuropathology data set, except where otherwise specified.

\section{Pathological diagnosis}

The 4419 participants were categorized based on diagnostic criteria for AD, DLB, frontotemporal lobar degeneration (FTD), medial temporal lobe sclerosis (MTLS), and other major pathological diagnoses as follow.

AD: If Thal phase was assessed, an "ABC" score indicating intermediate or high ADNC [11] constituted an $\mathrm{AD}$ diagnosis. Otherwise, pathological diagnosis of $\mathrm{AD}$ followed NIA-Reagan criteria (i.e., at least Consortium to Establish a Registry for Alzheimer's Disease (CERAD) moderate and Braak stage III/IV) [12].

DLB: Pathological diagnosis of DLB followed criteria outlined in the fourth consensus report of the DLB Consortium (i.e., requires limbic (transitional) or diffuse neocortical Lewy-related pathology) [14].

MTLS: MTLS (including hippocampal sclerosis) was determined based on pathologist report to the NACC as present or absent.

FTD and other tauopathies: Evidence of FTD with tau pathology (including Pick's disease, corticobasal degeneration, and progressive supranuclear palsy), FTD and parkinsonism with tau-positive or argyrophilic inclusions, other tauopathies (including tangle-only dementia and argyrophilic grain dementia), FTD with ubiquitin-positive (tau-negative) inclusions, FTD with TDP-43 pathology, and FTD with no distinctive histopathology present or not otherwise specified constituted an FTD pathological diagnosis.

Other Pathological Diagnoses: Cases with "other pathological diagnoses" were excluded. Specifically, in versions 1-9 of the NACC neuropathology data, this constituted evidence of prion-related disorders and other major pathologic disorders (e.g. infectious, immunologic, metabolic, neosplastic, toxic, or degenerative). In version 10 , this constituted ALS/motor neuron disease, Pigmentspheroid degeneration/NBIA, multiple system atrophy, prion disease, trinucleotide disease (Huntington disease, SCA, or other), malformation of cortical development, metabolic/storage disorder, leukodystrophy, multiple sclerosis or other demyelinating disease, contusion/traumatic brain injury of any type (acute or chronic), neoplasm (primary or metastatic), infectious process of any type (encephalitis, abscess, etc.), herniation (any site), or other pathologic diagnosis, Down syndrome, ADrelated genes (dominantly inherited), FTD related genes (dominantly inherited), or other known genetic mutation. Neuron loss in the substantia nigra was additionally considered except in the case of a DLB pathological diagnosis.

To disentangle the effects of genetic risk on pathology in light of frequently occurring co-pathology, we restricted analysis to individuals who met the above criteria for only AD $(n=1854)$, DLB $(n=57)$, or FTD $(n=65)$ without meeting criteria for any other pathological diagnosis, those who met the criteria for both $A D$ and DLB but no other pathological diagnosis (AD + DLB, $\mathrm{n}=455$ ), and those who met the criteria for both $\mathrm{AD}$ and MTLS but no other pathological diagnosis (AD + MTLS, $\mathrm{n}=182$ ). Also, individuals who did not meet criteria for any of the above pathological diagnoses were included (control, $\mathrm{n}=245$ ). This lead to the exclusion of 1561 participants based on pathological criteria, either with mixed pathology inconsistent with the above groups, or with pathology documented in a way that precluded categorization (e.g. LBs present in an unspecified region).

\section{Clinical diagnoses}

Clinical diagnosis was assessed at the final visit before death. Individuals in the control group (who did not meet criteria for any of the pathological diagnoses) were further limited to clinically normal participants without PD, resulting in the exclusion of 68 additional participants. For all other pathologically defined groups, no clinical criteria were imposed except to exclude individuals with PD dementia, following the one-year rule, excluding an additional 14 participants (1 DLB and $13 \mathrm{AD}+\mathrm{DLB}$ ), and those with a prior clinical PD diagnosis who did not meet pathological criteria for DLB, excluding an additional 63 participants (55 AD, $7 \mathrm{AD}+$ MTLS, and 1 FTD). 


\section{Genetic data}

Genetic data for UCSD ADRC participants was accessed through the NACC database or obtained locally. Alzheimer's disease Genetics Consortium (ADGC) data for participants evaluated at other ADRCs was accessed through the National Institute on Aging Genetics of Alzheimer's disease Data Storage Site (NIAGADS). For the ADRC, genetic data was preprocessed with PLINK to exclude samples with a missingness rate greater than $10 \%$ and to perform strand flips as necessary. Pre-imputation quality controls removed duplicate sites, non-singlenucleotide polymorphism (SNP) sites, monomorphic sites, and SNPs with a call rate $<90 \%$. The imputation was performed using the Michigan Imputation Server [3] with the HRC reference panel [13] (hg19). Post-imputation the data was filtered to exclude genotype calls with an estimated posterior genotype probability $<0.9$. For the NACC, imputed genetic data was downloaded directly from NIAGADS.

\section{AD polygenic hazard score calculation}

The PHS was calculated as described for all participants [4]. Briefly, potentially AD-associated SNPs were selected in the International Genomics of Alzheimer's Project (IGAP) cohort at $p<10^{-5}$. These SNPs were then integrated into a stepwise Cox proportional hazards model using a subset of the ADGC phase 1 genetic data, excluding individuals from the NACC, Alzheimer's Disease Neuroimaging Initiative (ADNI), and the Religious Orders Study and Rush Memory and Aging Project (ROSMAP). This stepwise procedure identified 31 SNPs that most improved the model prediction. The derived PHS was then validated in independent samples, including ADGC phase 2, NACC, ADNI, and ROSMAP. The PHS used in the current study was calculated for each participant as the vector product of that individual's genotype for the 31 SNPs and the corresponding parameter estimates from the ADGC phase 1 Cox proportional hazard model, in addition to the $A P O E$ effects.

\section{PD polygenic risk score calculation}

The PD PRS was calculated for each participant as the vector product of that individual's genotype for the 90 independent genome-wide significant variants identified by the most recent meta-analysis of PD genome-wide association study (GWAS) data and the corresponding parameter estimates using data from all available studies [15].

\section{DLB polygenic risk score calculation}

The DLB PRS was calculated as the vector product of that individual's genotype for the 5 independent genome-wide significant variants identified by the first DLB GWAS and the corresponding parameter estimates from the discovery stage [8]. To ensure there was no participant overlap between samples, the DLB PRS was only analyzed in the subset of 2282 participants in the present study who were assuredly not included in the DLB GWAS.

\section{Statistical analysis}

Binary logistic regression was used to examine the relationship between the PD PRS and a clinical diagnosis of PD, controlling for age at death and sex. Clinical and demographic differences between pathologically defined groups were examined using either Welch's two sample $t$ test or Pearson's Chi squared test as appropriate, corrected for multiple comparisons using the Benjamini-Hochberg procedure. $A P O E \& 4$ allele frequency was examined across pathologically defined groups using Pearson's Chi squared test. Pairwise comparisons between groups were corrected for multiple comparisons using the Benjamini-Hochberg procedure.

Multinomial logistic regression models were used to examine the relationship between the AD PHS, PD PRS, or DLB PRS and the pathological diagnosis group, controlling for age at death and sex, and Bonferroni corrected for three comparisons. Given that the $A P O E \varepsilon 4$ allele is a known risk factor for both $A D$ and DLB, multinomial logistic regression models were used to examine the relationship between either the AD PHS or DLB PRS without its $A P O E$ component weights and the pathological diagnosis group, controlling for age at death and sex.

Ordinal logistic regression models were used to examine the relationship between either the AD PHS, PD PRS, or DLB PRS and AD pathological outcome variables (i.e., Braak stage for neurofibrillary tangles or CERAD score for neuritic plaques), controlling for age at death and sex. Multicollinearity was evaluated. Brant's test was used to test the proportional odds assumption. Proportional odds models were used except in the case where the proportional odds assumption was violated, in which cases partial proportional odds models were used. Binary logistic regression models were used to examine the relationship between the AD PHS, PD PRS, or DLB PRS and the presence of at least limbic (transitional) Lewy-related pathology, controlling for age at death and sex. For each pathological outcome variable, results were Bonferroni corrected for three comparisons. To examine the effect of the AD PHS and DLB PRS on pathology beyond APOE, analyses were repeated using versions of the AD PHS or DLB PRS without its APOE component weights. 


\section{Results}

In all 4419 participants, regardless of pathological diagnosis, the PD PRS predicted a clinical diagnosis of $\mathrm{PD}$ (odds ratio $(\mathrm{OR})=1.52,95 \%$ confidence interval $(\mathrm{CI})=1.16-1.98, p=0.002) .2713$ participants were categorized into one of six pathologically defined groups: FTD $(\mathrm{n}=64), \mathrm{DLB}(\mathrm{n}=56), \mathrm{AD}+\mathrm{DLB}(\mathrm{n}=442), \mathrm{AD}$ $(n=1799)$, AD + MTLS $(n=175)$ or control $(n=177)$. Clinical and demographic characteristics of these pathologically defined groups are reported in Table 1. Notable in Table 1 is the following: the age at death in each pathologically defined group, with the exception of $A D+M T L S$, was younger than in the control group, more men were in the DLB and AD + DLB groups than in the other groups, all $\mathrm{AD}$ groups $(\mathrm{AD}, \mathrm{AD}+\mathrm{DLB}$, and $\mathrm{AD}+\mathrm{MTLS}$ ) had worse cognitive impairment than the FTD or DLB groups, and, within AD groups, those with mixed pathology (AD + DLB or AD + MTLS) had worse cognitive impairment than those with only AD.

$A P O E \varepsilon 4$ allele frequency was different between pathologically defined groups (overall $\chi^{2}=174.5, p<.001$, Table 2). Pairwise Chi squared tests revealed that

Table 1 Demographics and clinical characteristics split by pathological diagnosis group

\begin{tabular}{|c|c|c|c|c|c|c|}
\hline & \multicolumn{6}{|c|}{ Pathological diagnosis group } \\
\hline & Control & FTD & DLB & $A D+D L B$ & AD & $A D+M T L S$ \\
\hline Participants, N & 177 & 64 & 56 & 442 & 1799 & 175 \\
\hline Women, N (\%) & $94(53)$ & $25(39)$ & 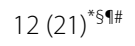 & $181(41)^{* \pi \#}$ & $959(53)$ & $94(54)$ \\
\hline Age at death, $y$ & $83.7(8.7)$ & $78.5(11.5)^{* \#}$ & $80.1(8.6)^{* \#}$ & $79.0(8.5)^{* \# \uparrow}$ & $80.2(9.0)^{* \#}$ & $84.6(7.8)$ \\
\hline Caucasian, N (\%) & $173(98)$ & $63(98)$ & $56(100)$ & $427(97)$ & $1736(96)$ & $171(98)$ \\
\hline Hispanic, N (\%) & $4(2)$ & $0(0)$ & $1(2)$ & $7(2)$ & $30(2)$ & $1(1)$ \\
\hline Education, y & $15.4(2.8)$ & $15.1(3.0)$ & $15.6(3.3)$ & $15.0(3.2)$ & $14.3(3.3)^{*} \neq \S$ & $14.8(3.6)$ \\
\hline \multicolumn{7}{|l|}{ Final clinical evaluation } \\
\hline Time before death, y & $2.6(3.0)$ & $3.0(3.0)$ & $2.9(3.5)$ & $3.0(3.3)$ & $2.5(2.8)^{\S \#}$ & $3.2(3.1)$ \\
\hline \multicolumn{7}{|c|}{ Primary clinical diagnosis } \\
\hline$A D, N(\%)$ & $0(0)$ & $22(34)$ & $19(34)$ & $340(77)$ & $1574(87)$ & $159(91)$ \\
\hline LBD, N (\%) & $0(0)$ & $2(3)$ & $24(43)$ & $79(18)$ & $41(2)$ & $4(2)$ \\
\hline FTD, N (\%) & $0(0)$ & $23(36)$ & $1(2)$ & $9(2)$ & $64(4)$ & $5(3)$ \\
\hline Global CDR & $0.1(0.2)$ & $1.6(1.2)^{*}$ & $1.4(1.0)^{*}$ & $2.1(0.9)^{*}+\neq$ & $1.9(1.0)^{*}+\ddagger \S \#$ & $2.2(0.8)^{*}+\ddagger$ \\
\hline CDR-SB & $0.1(0.4)$ & $9.0(6.8)^{*}$ & $7.6(5.8)^{*}$ & $12.5(5.5)^{*}+\neq$ & $10.8(6.1)^{*}+\ddagger \S \#$ & $12.9(4.9)^{*}+\ddagger$ \\
\hline
\end{tabular}

Reported as mean (SD) unless otherwise noted. Primary clinical diagnosis of AD included probable or possible AD, LBD included DLB, Lewy body variant of AD, and LBD, and FTD included FTD, Pick's disease, CBD, PSP, and PPA. FDR adjusted $p<.05$ for differences from *Control, †FTD, $\neq D L B, \S A D+D L B$, $1 A D$, or \#AD + MTLS based on pairwise Pearson's Chi squared tests or Welch's t-tests. Small numbers in certain subgroups prevented the pairwise comparison of primary clinical diagnoses across pathologically defined groups

Abbreviations: DLB, dementia with Lewy bodies; AD, Alzheimer's disease; FTD, frontotemporal lobar degeneration; MTLS, medial temporal lobe sclerosis; LBD, Lewy body dementia; CDR, Clinical Dementia Rating Scale; CDR-SB, Clinical Dementia Rating Scale Sum of Boxes

Table 2 APOE genotypes and allele frequencies by pathologically defined groups

\begin{tabular}{|c|c|c|c|c|c|c|c|}
\hline & \multicolumn{7}{|c|}{$\mathrm{N}(\%)$} \\
\hline & \multicolumn{6}{|c|}{$A P O E$ genotype frequency } & \multirow[t]{2}{*}{$\varepsilon 4$ allele frequency } \\
\hline & $2 / 2$ & $2 / 3$ & $2 / 4$ & $3 / 3$ & $3 / 4$ & $4 / 4$ & \\
\hline Control & $1(1)$ & $25(14)$ & $7(4)$ & $119(67)$ & $24(14)$ & $1(1)$ & $33(9)$ \\
\hline FTD & $0(0)$ & $7(11)$ & $1(2)$ & $37(58)$ & $19(30)$ & $0(0)$ & $20(16) \dagger$ \\
\hline DLB & $0(0)$ & $8(14)$ & $4(7)$ & $28(50)$ & $16(29)$ & $0(0)$ & $20(18)^{*} \dagger$ \\
\hline$A D+D L B$ & $2(0)$ & $11(2)$ & $5(1)$ & $136(31)$ & $224(51)$ & $64(14)$ & $357(40)^{*}$ \\
\hline$A D$ & $0(0)$ & $52(3)$ & $51(3)$ & $594(33)$ & $829(46)$ & $273(15)$ & $1426(40)^{*}$ \\
\hline$A D+M T L S$ & $0(0)$ & $8(5)$ & $5(3)$ & $58(33)$ & $83(47)$ & $21(12)$ & $130(37)^{*}$ \\
\hline
\end{tabular}

$A P O E$ \&4 allele frequency was different between pathologically defined groups (overall $x^{2}=174.5, p<.001$ ). FDR adjusted $p<.05$ for differences from ${ }^{*}$ Control, $+A$ Ill $A D$ groups (AD, $A D+D L B$, and $A D+M T L S)$ based on pairwise Chi squared tests

Abbreviations: DLB, dementia with Lewy bodies; AD, Alzheimer's disease; FTD, frontotemporal lobar degeneration; MTLS, medial temporal lobe sclerosis; APOE, apolipoprotein $\mathrm{E}$ 
$A P O E \varepsilon 4$ allele frequency was greater in all $\mathrm{AD}$ groups (AD, AD + DLB, and AD + MTLS) and the DLB group than in the control group (FDR adjusted $p<.05$ ). Additionally, $A P O E \& 4$ allele frequency was greater in all AD groups than in either the FTD or DLB groups (FDR adjusted $p<.001$ ).

Higher AD PHS was associated with increased relative risk ratios for the $A D, A D+D L B$, and $A D+M T L S$ pathological diagnosis groups, compared to the control group $(p<.001$, Table 3; Fig. 1$)$. Higher AD PHS was also associated with increased relative risk ratios for the DLB and FTD groups compared to the control group $(p<.01)$, though with lower relative risk ratios than any of the AD groups, as confirmed by non-overlapping confidence intervals. Higher PD PRS was not associated with significant increased relative risk ratios for any pathological group compared to the control group.
Higher DLB PRS was associated with increased relative risk ratios for the $\mathrm{AD}, \mathrm{AD}+\mathrm{DLB}, \mathrm{AD}+\mathrm{MTLS}$, and DLB groups compared to the control group $(p<.001)$.

Without the APOE $\varepsilon 4$ or $\varepsilon 2$ dosage weights, we observed similar results for the AD PHS, which was associated with increased relative risk ratios for the $\mathrm{AD}$ (1.86 95\% CI [1.47-2.37]), AD + DLB (1.83 [1.39-2.40]), AD+MTLS (1.91 [1.37-2.65]), DLB (2.03 [1.26-3.26]), and FTD (1.78 [1.14-2.78]) groups compared to the control group. Without the $A P O E$ component weight, the specificity of the DLB PRS emerged, as it was only associated with increased relative risk ratios for the DLB (3.58 [1.20-10.66]) and AD+DLB (3.15 [1.47-6.77]) groups compared to the control group.

The odds of having tau pathology at or above a given Braak stage increased with the AD PHS $(p<.001$, Table 3; Fig. 2). The PD PRS was not associated with Braak stage.

Table 3 Associations between the polygenic scores and pathological diagnostic categories and variables

\begin{tabular}{|c|c|c|c|c|c|c|}
\hline \multirow[t]{2}{*}{ Pathological diagnosis } & \multicolumn{2}{|l|}{ AD PHS } & \multicolumn{2}{|l|}{ PD PRS } & \multicolumn{2}{|l|}{ DLB PRS } \\
\hline & $\operatorname{RRR}(95 \% \mathrm{Cl})$ & pvalue & $\operatorname{RRR}(95 \% \mathrm{Cl})$ & $p$ value & $\operatorname{RRR}(95 \% \mathrm{Cl})$ & $p$ value \\
\hline \multicolumn{7}{|l|}{$M L R$, control as reference } \\
\hline FTD & $1.60(1.16-2.21)$ & .004 & $1.01(.60-1.70)$ & .97 & $1.24(.76-2.02)$ & .40 \\
\hline DLB & $1.73(1.23-2.42)$ & .002 & $1.39(.80-2.41)$ & .24 & $3.22(1.62-6.40)$ & $<.001$ \\
\hline$A D+D L B$ & $3.05(2.47-3.77)$ & $<.001$ & $.93(.67-1.28)$ & .65 & $4.47(2.76-7.22)$ & $<.001$ \\
\hline $\mathrm{AD}$ & $3.06(2.52-3.71)$ & $<.001$ & $.92(.69-1.22)$ & .57 & $3.08(2.33-4.09)$ & $<.001$ \\
\hline \multirow[t]{2}{*}{$A D+M T L S$} & $3.14(2.45-4.02)$ & $<.001$ & $.95(.65-1.39)$ & .78 & $2.94(2.07-4.18)$ & $<.001$ \\
\hline & OR $(95 \% \mathrm{Cl})$ & $p$ value & OR $(95 \% \mathrm{Cl})$ & pvalue & OR $(95 \% \mathrm{Cl})$ & pvalue \\
\hline \multicolumn{7}{|l|}{ Braak stage } \\
\hline OLR, POM & - & - & $0.93(0.82-1.05)$ & .24 & - & - \\
\hline \multicolumn{7}{|l|}{ OLR, PPOM } \\
\hline 1 & $2.34(1.60-3.42)$ & $<.001$ & - & - & $1.89(1.06-3.39)$ & .03 \\
\hline$\|$ & $2.10(1.76-2.50)$ & $<.001$ & - & - & $2.05(1.52-2.76)$ & $<.001$ \\
\hline III & $2.28(1.99-2.62)$ & $<.001$ & - & - & $2.33(1.88-2.89)$ & $<.001$ \\
\hline IV & $1.88(1.68-2.10)$ & $<.001$ & - & - & $1.95(1.63-2.33)$ & $<.001$ \\
\hline V & $1.53(1.40-1.68)$ & $<.001$ & - & - & $1.50(1.30-1.72)$ & $<.001$ \\
\hline $\mathrm{Vl}$ & $1.33(1.23-1.44)$ & $<.001$ & - & - & $1.34(1.18-1.51)$ & $<.001$ \\
\hline \multicolumn{7}{|l|}{ Neuritic plaque density } \\
\hline OLR, POM & - & - & $0.85(0.73-0.99)$ & .04 & - & - \\
\hline \multicolumn{7}{|l|}{ OLR, PPOM } \\
\hline Sparse & $2.69(2.21-3.26)$ & $<.001$ & - & - & $2.79(1.95-3.98)$ & $<.001$ \\
\hline Moderate & $2.42(2.07-2.84)$ & $<.001$ & - & - & $2.42(1.88-3.12)$ & $<.001$ \\
\hline Frequent & $1.71(1.55-1.88)$ & $<.001$ & - & - & $1.63(1.41-1.88)$ & $<.001$ \\
\hline \multicolumn{7}{|l|}{ Lewy pathology stage } \\
\hline BLR & $1.03(0.93-1.14)$ & .52 & $1.04(0.87-1.24)$ & .66 & $1.45(1.04-2.03)$ & .03 \\
\hline
\end{tabular}

Results of multinomial, ordinal, and binary logistic regression models for each polygenic score and pathological diagnosis group and outcome variable are displayed. For ordinal logistic regressions, proportional odds models were used except in the case where the proportional odds assumption was violated, in which cases partial proportional odds models were used. Effects are reported per unit increase in the polygenic score. Significance was set to $p<.017$, Bonferroni corrected for comparisons across the three polygenic scores

Abbreviations: RRR, relative risk ratio; OR, odds ratio; MLR, multinomial logistic regression; OLR, ordinal logistic regression; POM, proportional odds model; PPOM, partial proportional odds model; BLR, binary logistic regression PD, Parkinson's disease, PRS, polygenic risk score; DLB, dementia with Lewy bodies; AD, Alzheimer's disease; PHS, polygenic hazard score; FTD, frontotemporal lobar degeneration; MTLS, medial temporal lobe sclerosis 


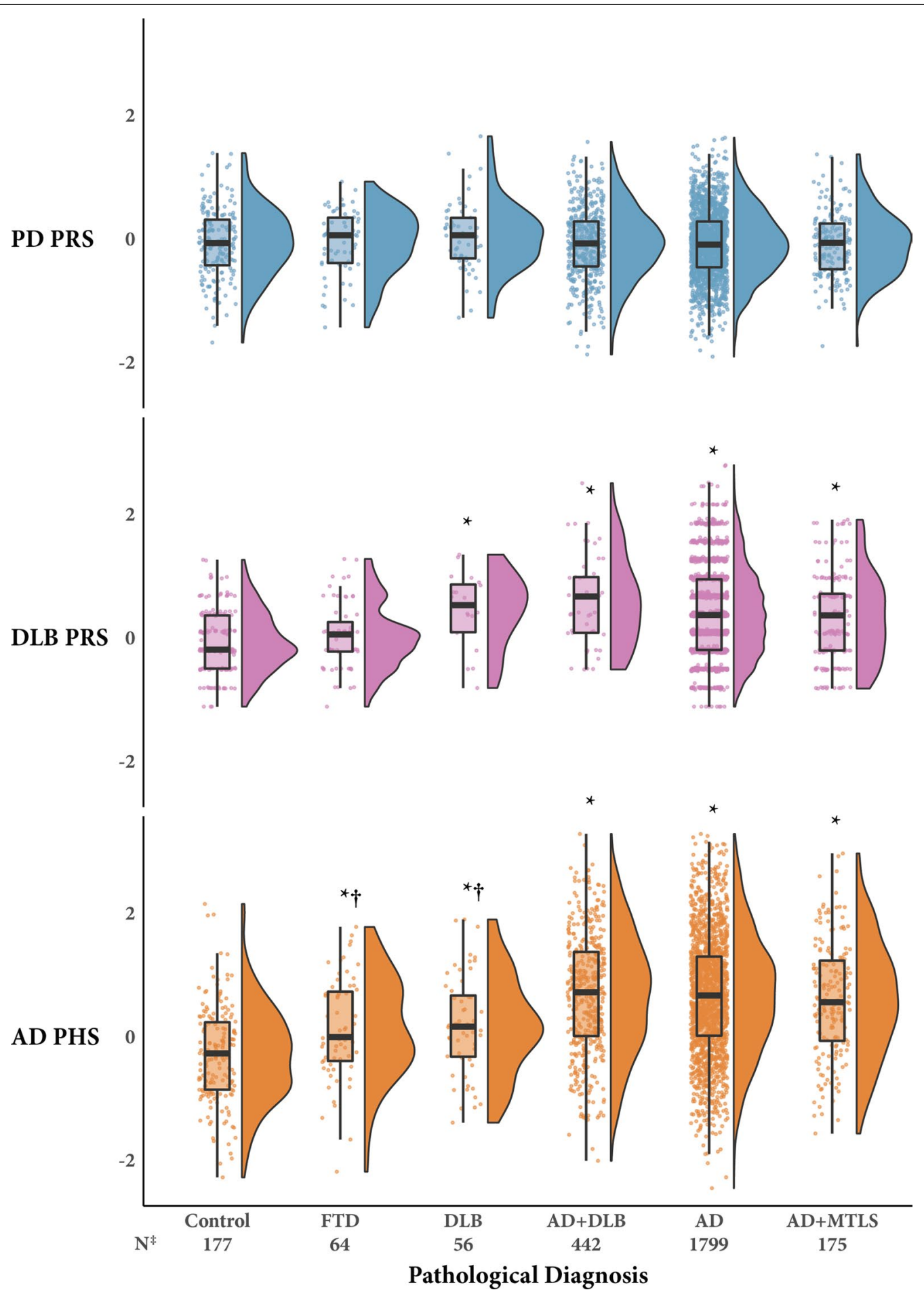

Fig. 1 Relationship between polygenic scores and pathological diagnostic categories. Significant difference from: *Control, $†$ All AD groups (AD, $A D+D L B$, and $A D+M T L S$ ) based on Bonferroni corrected multinomial regression models. $\neq$ The number of participants is reported for the $A D$ PHS and PD PRS analyses. The DLB PRS was analyzed in a subset of 2282 total participants. Abbreviations: PD, Parkinson's disease, PRS, polygenic risk score; DLB, dementia with Lewy bodies; AD, Alzheimer's disease; PHS, polygenic hazard score; FTD, frontotemporal lobar degeneration; MTLS, medial temporal lobe sclerosis 


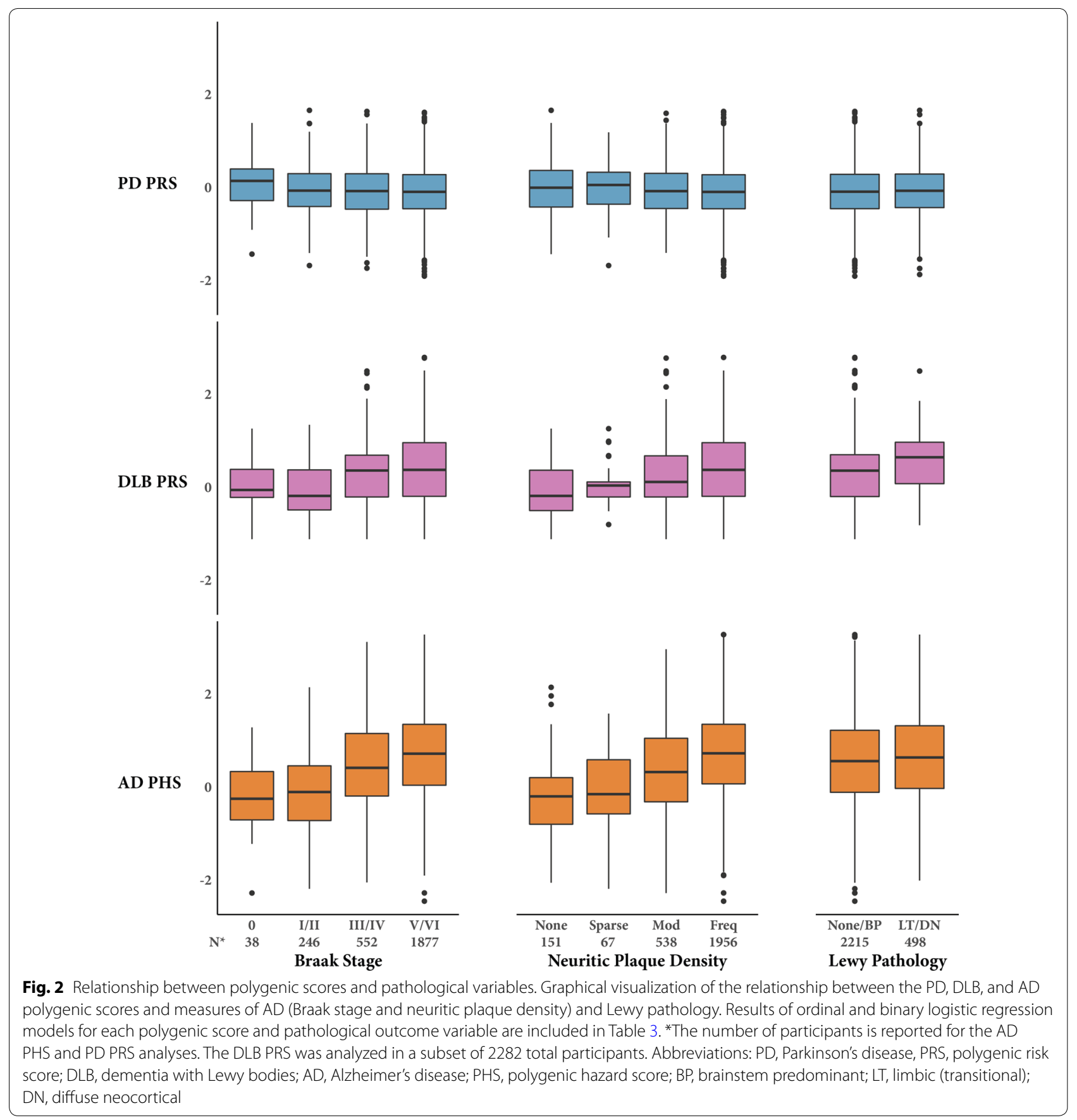

Beginning with Braak stage II, the odds of having tau pathology at or above a given Braak stage increased with DLB PRS $(p<.001)$. Without the APOE $\varepsilon 4$ or $\varepsilon 2$ dosage weights, we observed similar results for the association between Braak stage and the AD PHS (OR 1.20 95\% CI [1.08-1.34]). Yet, without the APOE component weight, the DLB PRS was not associated with Braak stage (0.89 [0.73-1.08]).
The odds of having neuritic plaques at or above a given density increased with both the AD PHS and DLB PRS $(p<.001$, Table 3; Fig. 2). The PD PRS was not associated with neuritic plaque density. Without the $A P O E \varepsilon 4$ or $\varepsilon 2$ dosage weights, we observed similar results for the association between the AD PHS and neuritic plaque density (OR 1.25 95\% CI [1.10-1.43]). Conversely, without the $A P O E$ component weight, the odds of having 
neuritic plaques at or above a given density decreased with increasing DLB PRS (OR 0.76 [0.60-0.96]).

None of the polygenic scores were associated with the presence of at least limbic (transitional) LB pathology (Table 3; Fig. 2). Similarly, without the APOE $\varepsilon 4$ or $\varepsilon 2$ dosage weights, the AD PHS was not associated with the presence of at least limbic (transitional) LB pathology (OR 1.04 95\% CI [0.89-1.22]). However, without the $A P O E$ component weight, the DLB PRS was associated with the odds of having at least limbic (transitional) LB pathology (3.41 [1.93-6.03]).

\section{Discussion}

We tested whether genetic risk for $\mathrm{AD}$, DLB, or PD may be useful to clinically distinguish between $A D$ and DLB. The AD and DLB polygenic scores were associated with their respective pathological diagnostic categories, though not exclusively. We replicated the finding of an overrepresentation of the APOE $\varepsilon 4$ allele in the pure DLB group compared to controls [24], but found that the $\varepsilon 4$ allele frequency was higher in all $\mathrm{AD}$ groups $(\mathrm{AD}$, $\mathrm{AD}+\mathrm{DLB}$, or AD + MTLS) than in the DLB group. Given this increased frequency and the strong, dose-dependent risk of the $\varepsilon 4$ allele in $\mathrm{AD}$, the inclusion of an $A P O E$ weight in the DLB PRS diminished its ability to specifically predict LB pathology. When the $A P O E$ weight was removed, the DLB PRS was associated with only DLB and $\mathrm{AD}+\mathrm{DLB}$ pathological diagnosis groups, and was associated with increased LB but not AD pathology.

However, the dose-dependent weighting of the $A P O E$ $\varepsilon 4$ allele is an important feature of the AD PHS, and enabled the distinction between AD groups and the nonAD groups. When removing the $A P O E \varepsilon 2$ and $\varepsilon 4$ dosage weights, the AD PHS maintained its association with Braak stage and neuritic plaque density, but it also maintained associations with all pathological groups relative to the control group. The AD PHS includes genes associated with multiple biological processes implicated in $\mathrm{AD}$, such as inflammation, synaptic function, and epigenetic regulation [10]. The associations suggest such processes may also be disrupted in non-AD dementias.

The PD PRS was associated with a clinical PD diagnosis, but not any pathological diagnosis or variable examined in this study. These results align with recent work finding genetic risk for PD explained only a small amount (.37\%) of variance in DLB [7].

Despite striking group-level results, one limitation to the clinical utility of this work is the amount of individual variation in polygenic scores within pathological groups. It remains unclear whether assessing polygenic risk for $\mathrm{AD}$ and DLB in combination with clinical features and biomarkers improves the accuracy of clinical diagnoses at the individual level. Further, the polygenic scores used in these analyses were predominantly derived and tested on individuals of European ancestry. Known differences in genetic risk across racial and ethnic groups [17, 18, 23] suggest these findings may not generalize. Future work is required to develop polygenic scores in diverse populations.

\section{Conclusions}

Despite few identified genome-wide significant variants, the DLB PRS without the APOE component weight was specifically associated with LB pathology and a pathological diagnosis of DLB, either alone or in combination with AD. The AD PHS was associated with ADNC but not LB pathology, and most strongly predicted a pathological diagnosis of $\mathrm{AD}$, either alone or in combination with DLB or MTLS. Together, these results and the lack of associations with the PD PRS align with evidence that genetic risk for DLB is not simply situated in the middle of an AD-PD continuum, but has a distinct signature that can be exploited along with risk for AD to improve clinical diagnoses.

\begin{abstract}
Abbreviations
AD: Alzheimer's disease; DLB: dementia with Lewy bodies; NC: neuropathologic changes; LB: Lewy body; PHS: polygenic hazard score; PD: Parkinson's disease; APOE: apolipoprotein E; PRS: polygenic risk score; ADRC: Alzheimer's Disease Research Center; UCSD: University of California, San Diego; NACC: National Alzheimer's Coordinating Center; FTD: frontotemporal lobar degeneration; MTLS: medial temporal lobe sclerosis; CERAD: Consortium to Establish a Registry for Alzheimer's Disease; ADGC: Alzheimer's disease Genetics Consortium; NIAGADS: National Institute on Aging Genetics of Alzheimer's disease Data Storage Site; SNPs: single-nucleotide polymorphisms; IGAP: International Genomics of Alzheimer's Project; GWAS: genome-wide association study; ADNC: Alzheimer's disease neuropathologic changes.
\end{abstract}

\section{Acknowledgements}

Not applicable.

\section{Authors' contributions}

All authors revised and approved the final manuscript. BES: Conception and design of work, data analysis and interpretation, drafting of the manuscript. RGJ: Design of work, data analysis and interpretation. CCF: Data acquisition and interpretation. JBB: Conception and design of work, data analysis and interpretation, drafting of the manuscript. All authors read and approved the final manuscript.

\section{Funding}

This research was supported by grant 1P30-AG062429-01 from the National Institutes of Health (University of California, San Diego Alzheimer's Disease Research Center). The NACC database is funded by NIA/NIH Grant U01 AG016976. NACC data are contributed by the NIA-funded ADCs: P30 AG019610 (PI Eric Reiman, MD), P30 AG013846 (PI Neil Kowall, MD), P30 AG062428-01 (PI James Leverenz, MD) P50 AG008702 (PI Scott Small, MD), P50 AG025688 (PI Allan Levey, MD, PhD), P50 AG047266 (PI Todd Golde, MD, PhD), P30 AG010133 (PI Andrew Saykin, PsyD), P50 AG005146 (PI Marilyn Albert, PhD), P30 AG062421-01 (PI Bradley Hyman, MD, PhD), P30 AG062422-01 (PI Ronald Petersen, MD, PhD), P50 AG005138 (PI Mary Sano, PhD), P30 AG008051 (PI Thomas Wisniewski, MD), P30 AG013854 (PI Robert Vassar, PhD), P30 AG008017 (PI Jeffrey Kaye, MD), P30 AG010161 (PI David Bennett, MD), P50 AG047366 (PI Victor Henderson, MD, MS), P30 AG010129 (PI Charles DeCarli, MD), P50 AG016573 (PI Frank LaFerla, PhD), P30 AG062429-01(PI James Brewer, 
MD, PhD), P50 AG023501 (PI Bruce Miller, MD), P30 AG035982 (PI Russell Swerdlow, MD), P30 AG028383 (PI Linda Van Eldik, PhD), P30 AG053760 (PI Henry Paulson, MD, PhD), P30 AG010124 (PI John Trojanowski, MD, PhD), P50 AG005133 (PI Oscar Lopez, MD), P50 AG005142 (PI Helena Chui, MD), P30 AG012300 (PI Roger Rosenberg, MD), P30 AG049638 (PI Suzanne Craft, PhD), P50 AG005136 (PI Thomas Grabowski, MD), P30 AG062715-01 (PI Sanjay Asthana, MD, FRCP), P50 AG005681 (PI John Morris, MD), P50 AG047270 (PI Stephen Strittmatter, MD, PhD). The National Institutes of Health, National Institute on Aging (NIH-NIA) supported this work through the following grants: ADGC, U01 AG032984, RC2 AG036528; NACC, U01 AG016976; NCRAD, U24 AG021886; NIA LOAD, U24 AG026395, U24 AG026390; Banner Sun Health Research Institute P30 AG019610; Boston University, P30 AG013846, U01 AG10483, R01 CA129769, R01 MH080295, R01 AG017173, R01 AG025259, R01AG33193; Columbia University, P50 AG008702, R37 AG015473; Duke University, P30 AG028377, AG05128; Emory University, AG025688; Group Health Research Institute, UO1 AG06781, UO1 HG004610; Indiana University, P30 AG10133; Johns Hopkins University, P50 AG005146, R01 AG020688; Massachusetts General Hospital, P50 AG005134; Mayo Clinic, P50 AG016574; Mount Sinai School of Medicine, P50 AG005138, P01 AG002219; New York University, P30 AG08051, MO1RR00096, UL1 RR029893, 5R01AG012101, 5R01AG022374, 5R01AG013616, 1RC2AG036502, 1R01AG035137; Northwestern University, P30 AG013854; Oregon Health \& Science University, P30 AG008017, R01 AG026916; Rush University, P30 AG010161, R01 AG019085, R01 AG15819, R01 AG17917, R01 AG30146; TGen, R01 NS059873; University of Alabama at Birmingham, P50 AG016582, UL1RR02777; University of Arizona, R01 AG031581; University of California, Davis, P30 AG010129; University of California, Irvine, P50 AG016573, P50, P50 AG016575, P50 AG016576, P50 AG016577; University of California, Los Angeles, P50 AG016570; University of California, San Diego, P50 AG005131; University of California, San Francisco, P50 AG023501, P01 AG019724; University of Kentucky, P30 AG028383, AG05144; University of Michigan, P50 AG008671; University of Pennsylvania, P30 AG010124; University of Pittsburgh, P50 AG005133, AG030653; University of Southern California, P50 AG005142; University of Texas Southwestern, P30 AG012300; University of Miami, R01 AG027944, AG010491, AG027944, AG021547, AG019757; University of Washington, P50 AG005136; Vanderbilt University, R01 AG019085; and Washington University, P50 AG005681, P01 AG03991. The Kathleen Price Bryan Brain Bank at Duke University Medical Center is funded by NINDS grant \# NS39764, NIMH MH60451 and by Glaxo Smith Kline. Genotyping of the TGEN2 cohort was supported by Kronos Science. The TGen series was also funded by NIA grant AG034504 to AJM, The Banner Alzheimer's Foundation, The Johnnie B. Byrd Sr. Alzheimer's Institute, the Medical Research Council, and the state of Arizona and also includes samples from the following sites: Newcastle Brain Tissue Resource (funding via the Medical Research Council, local NHS trusts and Newcastle University), MRC London Brain Bank for Neurodegenerative Diseases (funding via the Medical Research Council),South West Dementia Brain Bank (funding via numerous sources including the Higher Education Funding Council for England (HEFCE), Alzheimer's Research Trust (ART), BRACE as well as North Bristol NHS Trust Research and Innovation Department and DeNDRoN), The Netherlands Brain Bank (funding via numerous sources including Stichting MS Research, Brain Net Europe, Hersenstichting Nederland Breinbrekend Werk, International Parkinson Fonds, Internationale Stiching Alzheimer Onderzoek), Institut de Neuropatologia, Servei Anatomia Patologica, Universitat de Barcelona. ADNI Funding for ADNI is through the Northern California Institute for Research and Education by grants from Abbott, AstraZeneca AB, Bayer Schering Pharma AG, Bristol-Myers Squibb, Eisai Global Clinical Development, Elan Corporation, Genentech, GE Healthcare, GlaxoSmithKline, Innogenetics, Johnson and Johnson, Eli Lilly and Co., Medpace, Inc., Merck and Co., Inc., Novartis AG, Pfizer Inc, F. Hoffman-La Roche, Schering-Plough, Synarc, Inc., Alzheimer's Association, Alzheimer's Drug Discovery Foundation, the Dana Foundation, and by the National Institute of Biomedical Imaging and Bioengineering and NIA grants U01 AG024904, RC2 AG036535, K01 AG030514. We thank Drs. D. Stephen Snyder and Marilyn Miller from NIA who are ex-officio ADGC members. Support was also from the Alzheimer's Association (LAF, IIRG-08-89720; MP-V, IIRG-05-14147) and the US Department of Veterans Affairs Administration, Office of Research and Development, Biomedical Laboratory Research Program. P.S.G.-H. is supported by Wellcome Trust, Howard Hughes Medical Institute, and the Canadian Institute of Health Research. Data for this study were prepared, archived, and distributed by the National Institute on Aging Alzheimer's Disease Data Storage Site (NIAGADS) at the University of Pennsylvania (U24-AG041689-01), funded by the National Institute on Aging.

\section{Availability of data and materials}

The datasets supporting the conclusions of this article are available in the NACC (alz.washington.edu) or NIAGADS (niagads.org) repositories. A request for resources, materials, or participant referrals from the Shiley-Marcos ADRC can be made by emailing Christina Gigliotti, Ph.D at cgigliotti@ucsd.edu.

\section{Ethics approval and consent to participate}

For submission of data to the NACC, each local institutional review board approved the research protocol and written informed consent was obtained from each participant or participant's guardian.

\section{Consent for publication}

Not applicable.

\section{Competing interests}

Dr. Brewer has served on advisory boards for Elan, Bristol-Myers Squibb, Avanir, Novartis, Genentech, and Eli Lilly and holds stock options in CorTechs Labs, Inc. and Human Longevity, Inc.

\section{Author details \\ ${ }^{1}$ Department of Neurosciences, University of California, San Diego, 9500 Gilman Drive, Mail Code 0949, La Jolla, CA 92093, USA. ${ }^{2}$ Center for Human Development, University of California, San Diego, La Jolla, CA, USA. ${ }^{3}$ Depart- ment of Radiology, University of California, San Diego, La Jolla, CA, USA.}

\section{Received: 11 July 2020 Accepted: 29 August 2020}

Published online: 10 September 2020

\section{References}

1. Bras J, Guerreiro R, Darwent L, Parkkinen L, Ansorge O, Escott-Price V et al (2014) Genetic analysis implicates APOE, SNCA and suggests lysosomal dysfunction in the etiology of dementia with Lewy bodies. Hum Mol Genet 23:6139-6146. https://doi.org/10.1093/hmg/ddu334

2. Connor DJ, Salmon DP, Sandy TJ, Galasko D, Hansen LA, Thal LJ (1998) Cognitive profiles of autopsy-confirmed Lewy body variant vs pure Alzheimer disease. Arch Neurol 55:994-1000. https://doi.org/10.1001/archn eur.55.7.994

3. Das S, Forer L, Schönherr S, Sidore C, Locke AE, Kwong A et al (2016) Next-generation genotype imputation service and methods. Nat Genet 48:1284-1287. https://doi.org/10.1038/ng.3656

4. Desikan RS, Fan CC, Wang Y, Schork AJ, Cabral HJ, Cupples LA et al (2017) Genetic assessment of age-associated Alzheimer disease risk: development and validation of a polygenic hazard score. PLoS Med 14:e1002289. https://doi.org/10.1371/journal.pmed.1002258

5. Galasko D, Hansen LA, Katzman R, Wiederholt W, Masliah E, Terry R et al (1994) Clinical-neuropathological correlations in Alzheimer's disease and related dementias. Arch Neurol 51:888-895. https://doi.org/10.1001/ archneur.1994.00540210060013

6. Galasko D, Katzman R, Salmon DP, Hansen L (1996) Clinical and neuropathological findings in Lewy body dementias. Brain Cogn 31:166-175. https://doi.org/10.1006/brcg.1996.0040

7. Guerreiro R, Escott-Price V, Hernandez DG, Kun-Rodrigues C, Ross OA, Orme T et al (2019) Heritability and genetic variance of dementia with Lewy bodies. Neurobiol Dis 127:492-501. https://doi.org/10.1016/j. nbd.2019.04.004

8. Guerreiro R, Ross OA, Kun-Rodrigues C, Hernandez DG, Orme T, Eicher JD et al (2018) Investigating the genetic architecture of dementia with Lewy bodies: a two-stage genome-wide association study. Lancet Neurol 17:64-74. https://doi.org/10.1016/S1474-4422(17)30400-3

9. Hansen L, Salmon D, Galasko D, Masliah E, Katzman R, DeTeresa R et al (1990) The Lewy body variant of Alzheimer's disease: a clinical and pathologic entity. Neurology 40:1-8. https://doi.org/10.1212/wnl.40.1.1

10. Hara Y, McKeehan N, Fillit HM (2019) Translating the biology of aging into novel therapeutics for Alzheimer disease. Neurology 92:84-93. https:// doi.org/10.1212/WNL.0000000000006745

11. Hyman BT, Phelps CH, Beach TG, Bigio EH, Cairns NJ, Carrillo MC et al (2012) National Institute on Aging-Alzheimer's Association guidelines for the neuropathologic assessment of Alzheimer's disease. Alzheimers Dement 8:1-13. https://doi.org/10.1016/j.jalz.2011.10.007 
12. Hyman BT, Trojanowski JQ (1997) Consensus recommendations for the postmortem diagnosis of Alzheimer disease from the National Institute on Aging and the Reagan Institute Working Group on diagnostic criteria for the neuropathological assessment of Alzheimer disease. J Neuropathol Exp Neurol 56:1095-1097. https://doi.org/10.1097/00005 072-199710000-00002

13. McCarthy S, Das S, Kretzschmar W, Delaneau O, Wood AR, Teumer A et al (2016) A reference panel of 64,976 haplotypes for genotype imputation. Nat Genet 48:1279-1283. https://doi.org/10.1038/ng.3643

14. McKeith IG, Boeve BF, Dickson DW, Halliday G, Taylor J-P, Weintraub D et al (2017) Diagnosis and management of dementia with Lewy bodies: fourth consensus report of the DLB Consortium. Neurology 89:88-100. https:// doi.org/10.1212/WNL.0000000000004058

15. Nalls MA, Blauwendraat C, Vallerga $C L$, Heilbron K, Bandres-Ciga S, Chang $D$ et al (2019) Identification of novel risk loci, causal insights, and heritable risk for Parkinson's disease: a meta-analysis of genome-wide association studies. Lancet Neurol 18:1091-1102. https://doi.org/10.1016/S1474 -4422(19)30320-5

16. Nalls MA, Duran R, Lopez G, Kurzawa-Akanbi M, McKeith IG, Chinnery PF et al (2013) A multicenter study of glucocerebrosidase mutations in dementia with Lewy bodies. JAMA Neurol 70:727-735. https://doi. org/10.1001/jamaneurol.2013.1925

17. Reitz C, Jun G, Naj A, Rajbhandary R, Vardarajan BN, Wang L-S et al (2013) Variants in the ATP-binding cassette transporter (ABCA7), apolipoprotein $E \in 4$, and the Risk of late-onset Alzheimer disease in African Americans. JAMA 309:1483-1492. https://doi.org/10.1001/jama.2013.2973

18. Romas SN, Santana V, Williamson J, Ciappa A, Lee JH, Rondon HZ et al (2002) Familial Alzheimer disease among caribbean hispanics: a reexamination of its association with APOE. Arch Neurol 59:87-91. https://doi. org/10.1001/archneur.59.1.87
19. Rongve A, Witoelar A, Ruiz A, Athanasiu L, Abdelnour C, Clarimon J et al (2019) GBA and APOE $\varepsilon 4$ associate with sporadic dementia with Lewy bodies in European genome wide association study. Sci Rep 9:7013. https ://doi.org/10.1038/s41598-019-43458-2

20. Schneider JA, Arvanitakis Z, Bang W, Bennett DA (2007) Mixed brain pathologies account for most dementia cases in community-dwelling older persons. Neurology 69:2197-2204. https://doi.org/10.1212/01. wnl.0000271090.28148.24

21. Stern Y, Jacobs D, Goldman J, Gomez-Tortosa E, Hyman BT, Liu Y et al (2001) An investigation of clinical correlates of Lewy bodies in autopsy-proven Alzheimer disease. Arch Neurol 58:460-465. https://doi. org/10.1001/archneur.58.3.460

22. Tan CH, Bonham LW, Fan CC, Mormino EC, Sugrue LP, Broce IJ et al (2019) Polygenic hazard score, amyloid deposition and Alzheimer's neurodegeneration. Brain 142:460-470. https://doi.org/10.1093/brain/awy327

23. Tang M-X, Stern Y, Marder K, Bell K, Gurland B, Lantigua R et al (1998) The APOE- $\varepsilon 4$ allele and the risk of Alzheimer disease among African Americans, Whites, and Hispanics. JAMA 279:751-755. https://doi.org/10.1001/ jama.279.10.751

24. Tsuang D, Leverenz JB, Lopez OL, Hamilton RL, Bennett DA, Schneider JA et al (2013) APOE \&4 increases risk for dementia in pure synucleinopathies. JAMA Neurol 70:223-228. https://doi.org/10.1001/jamaneurol .2013 .600

25. Walker Z, Possin KL, Boeve BF, Aarsland D (2015) Lewy body dementias. Lancet 386:1683-1697. https://doi.org/10.1016/S0140-6736(15)00462-6

\section{Publisher's Note}

Springer Nature remains neutral with regard to jurisdictional claims in published maps and institutional affiliations.
Ready to submit your research? Choose BMC and benefit from:

- fast, convenient online submission

- thorough peer review by experienced researchers in your field

- rapid publication on acceptance

- support for research data, including large and complex data types

- gold Open Access which fosters wider collaboration and increased citations

- maximum visibility for your research: over $100 \mathrm{M}$ website views per year

At BMC, research is always in progress.

Learn more biomedcentral.com/submissions 